\title{
Horn Operational Experience in K2K, MiniBooNE, NuMI and CNGS
}

\author{
Ans Pardons ${ }^{1}$ \\ CERN \\ Geneva, Switzerland \\ E-mail: ans.pardons@cern.ch
}

This paper gives an overview of the operation and experience gained in the running of magnetic horns in conventional neutrino beam lines (K2K, MiniBooNE, NuMI and CNGS) over the last decade. Increasing beam power puts higher demands on horn conductors but even more on their hydraulic and electrical systems, while the horn environment itself becomes more hostile due to radiation. Experience shows that designing horns for remote handling and testing them extensively without beam become prerequisites for successful future neutrino beam lines.

10th International Workshop on Neutrino Factories, Super beams and Beta beams Valencia, Spain

30 June - 05 July, 2008

\footnotetext{
$1 \quad$ Speaker
} 


\section{Introduction}

The present-generation conventional neutrino beam lines use one or more magnetic horns to focus the charged particles emitted from the target (Figure 1).

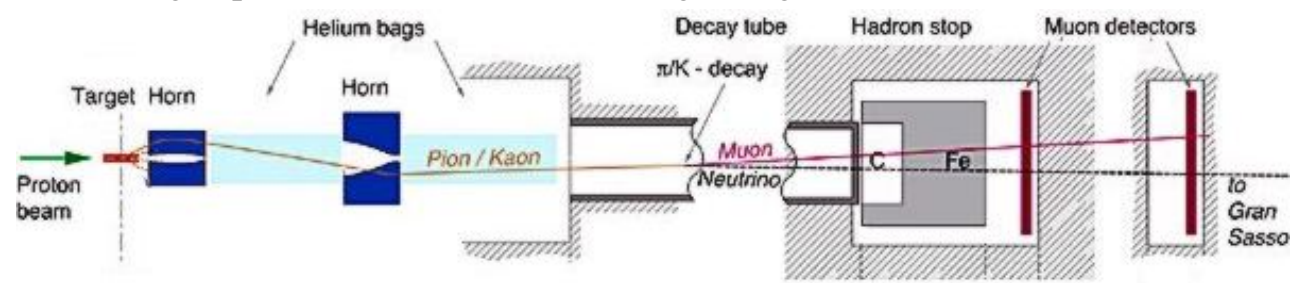

Figure 1: Main components of the CNGS neutrino beam line, with two magnetic horns.

A horn is a high-current, pulsed focusing device aimed at increasing the intensity and selecting the energy of a neutrino beam. A modern horn (Figure 2a-c) consists of a water-cooled aluminium inner and outer conductor, connected to a power source by parallel busbar plates.
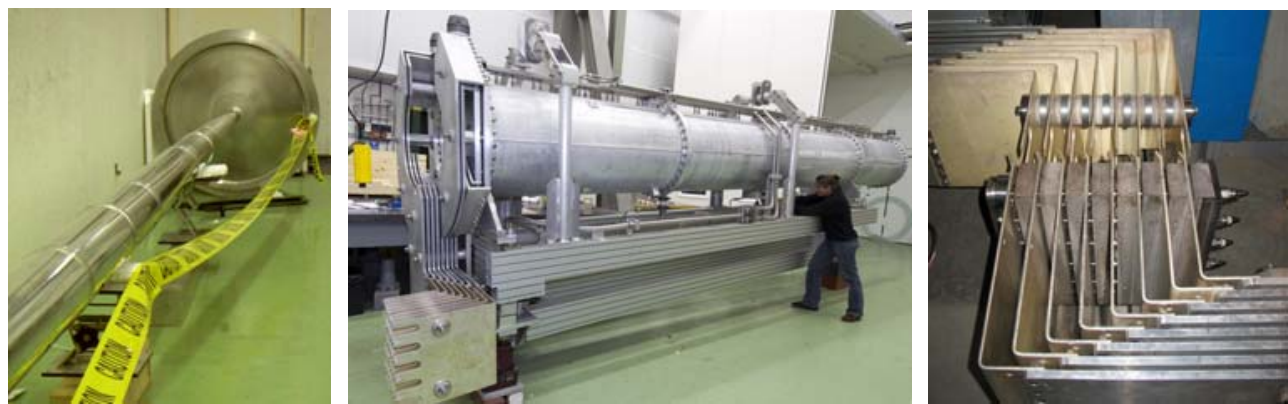

Figures 2a-c: CNGS horn inner conductor, assembled horn and horn-busbar connection.

Table 1 lists the main beam and horn characteristics of neutrino beams. Both inner and outer conductors, as well as the busbars see large current-induced cyclic stresses from the Joule heat load and from magnetic forces between conductors. The heat load from radiation, caused by particles traversing the inner conductor, causes an additional stress. The forces and vibrations are transmitted to the hydraulic and electrical systems, where they can cause fatigue failure.

When the beam power increases, the extra radiation-induced heat load causes additional stress. Also the horn environment becomes substantially more radioactive. This puts more constraints on the materials and makes in-situ repair extremely difficult or even impossible.

\begin{tabular}{l|llll|}
\hline & $\begin{array}{l}\text { K2K } \\
\mathbf{( 1 9 9 9 - 2 0 0 4 )}\end{array}$ & $\begin{array}{l}\text { MiniBoone } \\
\text { (since 2002) }\end{array}$ & $\begin{array}{l}\text { NuMI } \\
\text { (since 2004) }\end{array}$ & $\begin{array}{l}\text { CNGS } \\
\text { (since 2006) }\end{array}$ \\
\hline Average Beam Power (kW) & 6 & 32 & 290 & 460 \\
\hline Number of Protons per Pulse & $6.10^{12}$ & $5.10^{12}$ & $3.10^{13}$ & $2.4 .10^{13}$ \\
Beam Energy (GeV) & 12 & 8 & 120 & 400 \\
Avg. PulsingFrequency (Hz) & 0.5 & 5 & 0.5 & 0.3 \\
Horn Pulsing Current (kA) & 250 & 170 & 200 & $150 / 180$ \\
Horn Design Lifetime (pulses) & $10^{7}$ & $10^{8}$ & $10^{7}$ & $4.10^{7}$ \\
Number of Horns in System & 2 & 1 & 2 & 2 \\
\hline
\end{tabular}

Table 1: Main characteristics of neutrino beams and their horn system. 


\section{2. $\mathbf{K} 2 \mathrm{~K}$}

Because of the relatively low beam power and consequently low radiation levels, K2K horns were not designed to be handled or exchanged remotely.

\section{$2.1 \quad$ Operation}

During its five years of operation, the K2K beam line saw two major horn failures, both in the first year. The first was a leak in a cracked weld on the straight water inlet pipes, induced by fatigue stress from cyclic thermal expansion of the horn outer conductor to which they were attached. Figure 3a shows the new semi-flexible pipes that were installed on all following horns to absorb the thermal expansion. The second failure occurred in a semi-flexible busbar connection consisting of twisted copper cables, where strong magnetic forces between conductors of different polarities deformed the cables (Figure 3b) and caused a short circuit. The cables were successfully replaced with more rigid plates as shown in Figure 3c.

Horns were exchanged preventively every year, as long as the radiation level in the horn area allowed it. In summer 2004, radiation levels in the area made the exchange impossible. Three months later, a horn inner conductor broke from fatigue, defining the end of K2K.
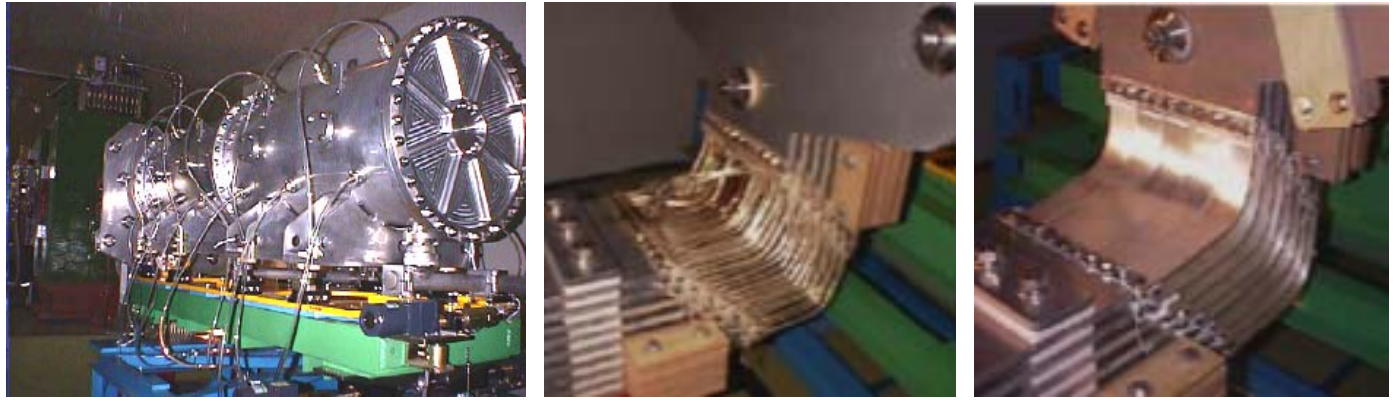

Figures 3a-c: K2K horn new water lines, faulty and new horn-busbar connection.

\subsection{Lessons learnt}

- Protect water input lines from vibrations of the outer conductor.

- Take magnetic forces into account when designing busbars and other conductors.

- Design future horn for remote exchange.

\section{MiniBooNE}

To maximize the lifetime of the MiniBooNE horn, material grades were carefully selected using restated fatigue data, while plating and anodizing were kept to a minimum. Learning from the K2K experience, the water input lines are installed on a vibration-insulated frame.

\subsection{Operation}

After 18 months of smooth running, water was seen leaking out of the ventilation box that encloses the entire horn, leading to a major ground fault that stopped the horn and beam line. Borescope images pointed towards galvanic corrosion of an aluminium seal between stainless steel flanges in a small volume of trapped water. 
The faulty horn was remotely replaced with a re-designed spare horn. In the new horn, the amount of trapped water is reduced through drainage and several aluminium seal connections are replaced with welded connections. This horn is still running in 2008 after a record 2.108 cycles.

\subsection{Lessons learnt}

- Avoid volumes with trapped water and carefully chose adjacent materials.

- Invest in a camera system or a shielded work cell as investigation of failures in-situ becomes harder as beam power increases.

\section{NuMI}

The two NuMI horns are designed for remote handling and exchange. A shielded and equipped work cell exists on top of the target hall shielding and permits observation of failures and limited repair interventions on a horn.

\subsection{Operation}

After a year of smooth running, both NuMI horns started to show several water leaks. All were due to the failure of off-the-shelf brazed ceramic-kovar assemblies, used as electrically insulated transitions in the water lines. By June 2008, about half of all assemblies had failed in the horns and all were replaced with an in-house developed brazing-free shrink-fit solution.

While replacement of the first assemblies and preventive replacements on the second (most downstream) horn could still be done in the work cell, the radiation around the first horn was too high to replace the last failed connection. This led to the exchange of the entire first horn with an enhanced spare. Since then, with all assemblies exchanged with the in-house solution, no further leaks have occurred.

\subsection{Lessons learnt}

- Beware of off-the-shelf solutions, especially when water and brazing are involved.

- Test horn and peripherals rigorously without beam, most failures happen early on.

\section{CNGS}

As is the case for NuMI, the CNGS horns are designed for remote handling and exchange. Special effort is put into the optimisation of dedicated tooling and in-situ practise before beam, minimizing the time and radiation dose taken by personnel in case of a future horn exchange.

\section{$5.1 \quad$ Operation}

After half a million pulses and a month of beam, a water leak appeared in the second (most downstream) horn's cooling circuit. One of the horn's electrically insulated brazed water outlets had a broken ceramic muff, as visible on Figure 4a. Analysis shows that the thin ceramic muff was weakened from machining and brazing. As a consequence, the relatively small forces from the horn pulsing sufficed to break the ceramic. A brazing-free alternative, where leak tightness 
is ensured by pressing soft graphite seals on a ceramic ring (Figure 4b-c), was installed. All brazed assemblies (outlets and inlets) were preventively replaced on both horns in a makeshift shielded area upstream of the target (Figure 4d). To reduce the personnel radiation dose, the intervention was carefully optimized and practised beforehand on the spare horn.

At the same time, a small fracture was discovered in one of the cables and some strands of the semi-flexible connection between busbar and transformer in the technical gallery. Since the magnetic forces seemed to have broken the brazed section of the cable, all cables were replaced with more solid plates, clamped together by insulators to better absorb the magnetic forces.
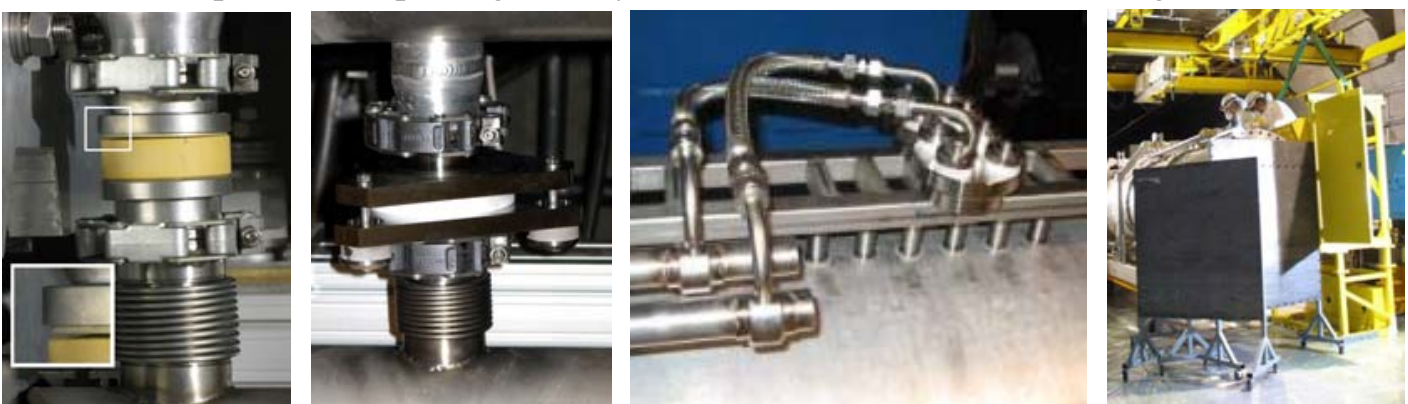

Figures 4 a-d: Faulty and new water outlet, new water inlets and in-situ intervention.

\section{$5.2 \quad$ Lessons learnt}

- Avoid brazing in the ceramic insulating assemblies of the cooling circuit.

- Invest in tooling, training and local shielding in case of local interventions.

\section{Conclusions}

Neutrino beams have used magnetic horns for 40 years to great success. However, with increasing beam power, the horns and their accessory systems must be enhanced to cope with the higher radiation, electrical heat load and mechanical forces. Operation experience from present-generation neutrino beams (K2K, MiniBooNE, NuMI and CNGS) shows that, while horn conductors can be designed to last an acceptably long life time, similar care must now be put into the design of the hydraulic and electrical accessory systems. Thanks to the Neutrino Beam \& Instrumentation workshops (NBI) and continuous exchange between the different laboratories, lessons learnt from the past do result in more reliable horns and accessory systems.

The horn environment becomes more radioactive with increasing beam power. This implies that in-situ repair is restricted to the first months of operation, as high radiation levels preclude interventions passed this initial period. Testing of the horn and all the accessory systems in-situ before beam becomes paramount to ensure a high uptime of the neutrino beam line. Remote horn handling and remote horn exchange have to be integrated in the design of the horn as well as the beam facility early on.

\section{Acknowledgements}

I wish to thank Larry Bartoszek, Ilias Efthymiopoulos, Edda Gschwendtner, Jim Hylen, Stephane Rangod and Kazuhiro Tanaka for their valuable input and useful discussions. I thank the technicians of my team at CERN for their work in putting the CNGS horns back on track. 